\title{
Ionic current enhancement through localised membrane geometrical deformation
}

\author{
Man Ting Kwong ${ }^{1}$, Antoine Jérusalem ${ }^{1 *}$
}

${ }^{1}$ Department of Engineering Science, University of Oxford, Parks Road, Oxford OX1 3PJ, UK

\author{
*Corresponding author \\ Professor Antoine Jérusalem \\ Address: Department of Engineering Science, University of Oxford, Parks Road, Oxford OX1 3PJ, UK \\ Email: antoine.jerusalem@eng.ox.ac.uk
}

\begin{abstract}
Bleb-inducing aspiration in a patch-clamp has previously been proposed as a controlled proxy for ion channel damage characterisation in brain trauma. Under increasing pressure, voltage gated sodium channels Nav1.6 have been observed to exhibit an altered dynamics known as "left-shift". While many numerical models have since been proposed to simulate this behaviour through modification of the ion channel dynamics in the Hodgkin-Huxley model, the effect of the deformed membrane geometry on the electrophysiology has not been explored. Here we reproduce in silico an idealised voltage clamp under the range of pressure used in its in vitro counterparts. The findings show that the geometrical alteration of the membrane within the pipette directly affects the patch electrophysiological measurements without requiring the ion channels to be damaged. This work highlights the need to consider geometrical deformation of the membrane when studying ion channel dynamics in deformed membrane and offers alternative explanations on the effect of blebbing on cellular and brain functions. It also opens the door to the possibility that membrane shape regulation and ionic signal enhancement can act independently with the former influencing the latter in neuronal migration and electrophysiological activity.
\end{abstract}

Keywords: patch-clamp, left-shift, Hodgkin-Huxley, finite element method, electrophysiologicalmechanical coupling 
Patch clamping is an experimental technique, where a patch of cell membrane is drawn into a pipette and its electrophysiology is measured by use of a stimulating electrode within the pipette. In a voltage clamp technique, the electrode inside the pipette is used to stabilise the membrane potential at a fixed voltage, while simultaneously recording the current that flows within the pipette. In particular, when the membrane is not ruptured ("cell attached" configuration), this technique is measuring the electrophysiology of a small number of ion channels within the patch (as opposed to "whole cell" configuration where the membrane inside the pipette is ruptured to record ion channels currents for the entire cell) and the measured feedback current is directly related to their activity.

Cell attached voltage clamp was used by Wang et al. to probe the channel dynamics of Nav1.6 in human embryonic kidney cells post-membrane trauma. ${ }^{1}$ Gigaseal (a seal formed between the cell membrane and the pipette wall, reaching resistance in the gigaohms range ${ }^{2}$ ) was achieved by "pre-stretching" patches at suction pressures between -4 and $-7 \mathrm{mmHg}$. Electrophysiological measurements in this configuration were taken as the reference. Subsequently, increasingly higher pressures were applied to the patch, with electrophysiological measurements reported to cause faster rise times in the voltage gated sodium channels Nav1.6 gating dynamics: the so-called "left-shift". ${ }^{1}$ Wang et al. hypothesised that direct membrane stretch through pipette suction causes Nav1.6 to become "leaky", suggesting that the channels are susceptible to trauma. This work has since motivated the development of many models built on the alteration of the ion channel dynamics in the Hodgkin-Huxley model. ${ }^{3-6}$

Cell membranes are known to deform under applied pressures similarly to two-dimensional fluids, ${ }^{7,8}$ effectively damping an overall membrane deformation to a fraction of its strain at the transmembrane protein level when the protein is untethered, both from the cytoskeleton and the extracellular matrix. ${ }^{9}$ This and the absence of evidence that the electrophysiology cannot be influenced by an alteration of the shape of the membrane patch call into question whether the observed "left-shift" can also occur in the absence of ion channel damage. To this end, a "pre-stretched" membrane patch geometry was modelled in silico by means of a finite element program to simulate the effect of subsequent pipette pressure on the patch electrophysiology. 
The cell geometry within the pipette was represented by a frustum of a cone (with a height of $2 \mu \mathrm{m}$, and upper and lower radii of 0.75 and $0.5 \mu \mathrm{m}$, respectively) and the main cell body immediately beneath the pipette was represented by a $3 \times 1 \times 3 \mu \mathrm{m}^{3}$ rectangular parallelepiped, see Figure 1 . The resulting geometry was discretised with 4,563 quadratic tetrahedral elements. A pressure boundary condition of $-50 \mathrm{mmHg}$ was applied to the top face of the frustum to simulate the suction applied by the pipette, see Figure 1a. The pipette walls and free surfaces of the box domain below were fixed in displacements. Since the electrophysiological-mechanical coupling is solely linked to the geometrical deformation of the membrane (see next paragraph), a linear elastic material law with a Young's modulus, density and Poisson's ratio of $165 \mathrm{kPa}, 993 \mathrm{~kg} \mathrm{~m}^{-3}$ and 0.3 , respectively, ${ }^{6}$ was considered adequate. In the simulations, the applied pressure was kept during action potential (AP) propagation, thus fixing the membrane geometry during the entire electrophysiological measurement. Membrane creep during patch clamp experiments has been previously observed, ${ }^{8}$ however the time scale of the creep is more than an order of magnitude larger than the time scale of an AP. Furthermore, this study focusses on the electrophysiology propagation on different static geometries arising from a pressure of $-50 \mathrm{mmHg}$, therefore a fixed deformed geometry was deemed acceptable.

The open source finite element platform $\mathrm{Gmsh}^{10}$ enriched with a dedicated electrophysiologicalmechanical solver ${ }^{6}$ was used to solve the finite element problem. In this novel approach, the active Hodgkin-Huxley electrophysiological model is imposed directly in the weak form as a Neumann boundary condition on the conductive three-dimensional domain. See Ref. (6) for more details, and in particular, for the values of the model parameters and their expressions in terms of patch area and other geometrical properties. In the work proposed here, regular Hodgkin-Huxley boundary conditions were considered for the membrane surrounding the pipette while the cell attached voltage-clamp at the top face of the frustum (inside the pipette) was modelled through a modified Hodgkin-Huxley boundary condition to include an external voltage of $20 \mathrm{mV}$ for the entire duration (12 ms) of the simulation so as to trigger an AP in the blebbed membrane within the pipette. The overall model thus remains the same as in Ref. (6), except for the following modification in the Hodgkin-Huxley equation where the term $\Delta \mathrm{V}=20 \mathrm{mV}$ is added: 
$i_{n}=c_{m} \frac{\partial V}{\partial t}+g_{N a}\left(V-E_{N a}+\Delta V\right)+g_{K}\left(V-E_{K}+\Delta V\right)+g_{L}\left(V-E_{L}+\Delta V\right)$

where $i_{n}, c_{m}, g_{N a}, g_{K}, g L, E_{N a}, E_{K}$ and $E_{L}$ are the outward current to the membrane, the membrane capacitance (for a given area), its $\mathrm{Na}_{\mathrm{v}}$ ion channel, $\mathrm{K}_{\mathrm{v}}$ ion channel and loss conductances, and the corresponding reversal potentials, respectively. Even after deformation, $\mathrm{Na}_{\mathrm{v}}$ and voltage gated potassium channel $\mathrm{K}_{\mathrm{V}}$ conductances were calculated with respect to the undeformed configuration, i.e., the current conductance associated to these channels remained the same throughout the deformation and independently of the area increase of the membrane (said otherwise, the number of ion channels in the top surface of the frustum remains the same). On the other hand, the leak and membrane resistances and capacitances were calculated with respect to the deformed reference frame and thus vary with the increase of surface area. The domain representing the gigasealed membrane (where the membrane is in contact with the pipette) was set to have no electrical flux across it, while the distal outer faces of the cytoplasm away from the pipette were free of flow. The Poisson's equation was chosen to govern the charge distribution in the bulk of the cytoplasm with a cytoplasm resistivity of $1.87 \Omega \mathrm{m} .^{6}$

Figures $1 \mathrm{a}$ and $1 \mathrm{~b}$ show the resultant deformation field at $5.65 \mathrm{~ms}$ and the post-suction intracellular voltage gradient (plotted in the undeformed configuration), respectively, while Figure 1c shows for comparison the voltage gradient at the same time $(5.65 \mathrm{~ms})$ when no suction is applied. Figure 2 shows the time evolution of the overall membrane current outflow in the pipette for both cases, and the respective (shifted) current rise tangents. These results indicate that: i) the geometry of the bleb has a strong influence on the current in the pipette (Figure 1b), and ii) the model is able to simulate some of the characteristics of a "left-shift" behaviour after aspiration without altering the ion channel dynamics, i.e., larger current amplitude and faster rise.

As the area of the membrane increases under negative pressure, a higher overall conductance across the membrane, and thus a faster rise for channel activation can indeed be expected. However, the overall conductance of a cytoplasmic element can either decrease or increase with the deformation, depending whether the current needs to go through more or less material over a larger or smaller area, see Figure 
3. This delicate balance between gain and loss in electrophysiological activity comes off here as an increase of overall current.

It must be emphasised that the magnitude of current in the above simulations is an order of magnitude smaller than the ones reported in the experiments. A simple reason for the discrepancy is the fact that the original Hodgkin-Huxley model was used in this model as a first approximation. In the experiments, a different cell was used and the ion channels were artificially transfected, making the identification of exact model to be used for the electrophysiology challenging. ${ }^{1}$ However, as long as a similar sodium ion channel behaviour is expected, the model's results are expected to qualitatively hold. Finally, the experiments were conducted on cells with $\mathrm{Na}_{\mathrm{v}} 1.6$ with and without auxiliary $\beta 1$ subunit (known to play a role in mechanotransduction behaviour ${ }^{11}$ ) with no marked resulting difference reported between the two, indicating that the reported effect may not be of a mechanosensitive nature. While this a priori seems to reinforce the experimental conclusion that the ion channels dynamics are altered through direct damage, this is also in agreement with the prediction of this work that the membrane deformation itself might be a vector for similar "left-shift" behaviour, independently of the ion channels. Finally, the results of Wang et al. exhibiting a given degree of irreversibility in the alteration of the electrophysiological behaviour ${ }^{1}$ are compatible with the notion of viscoelastic behaviour of the cell during suction, whereby the irreversible geometrical deformation of the membrane would invariably lead to an irreversible electrophysiological alteration in the proposed model. This phenomenon could thus readily be implemented with the right material constitutive model.

The above simulations demonstrate that geometrical alterations can alter the electrophysiological conduction in terms of a decreased rise time of channel activation, increase of current amplitude and signal delay, consistent with the experimentally observed "left-shift", but without the need to explicitly alter the channel gating dynamics. Wang et al.'s conclusions suggested that the "left-shift" dynamics could be justified by an increase in channel opening probability or area, as this would solely result in an increase in current amplitude and that the data thus pointed to trauma-induced $\mathrm{Na}_{\mathrm{v}} 1.6$ dynamics changes ${ }^{1}$. We demonstrate here that, in theory, "left-shift" characteristics can be observed without inducing ion channel damage through the additional consideration of the neighbouring deformed 
membrane. When widening the conclusions of this work to other channels, this result has direct implications on many brain functions.

Neuronal migration during brain development involves a complex coordination of cytoskeletal alterations (involving, among others, actin filaments, microtubules, myosin). ${ }^{12}$ In particular the distal end of the axon, the growth cone, is actively reshaping itself using motile filipodia and lamellipodia to sense its immediate environment. This sensing is done, among others, by use of transient receptor potential channels (TRP) permeable to $\mathrm{Ca}^{2+} \cdot{ }^{13}$ While TRP channels seem to be involved in mechanosensitive gating mechanisms, their exact involvements in neuronal migration is still under investigation. ${ }^{14}$ The result of this work suggests however that a radical change of membrane geometry is enough to affect the behaviour of ionic exchange with the cytoplasm. It is thus possible that the remodelling of the growth cone through $\mathrm{F}$-actin and integrin activities could indirectly affect TRP gating related $\mathrm{Ca}^{2+}$ influx by membrane remodelling. This hypothesis is of particular relevance to the debate over whether blebbing or $\mathrm{Ca}^{2+}$ influx (commonly observed conjointly) is the cause or consequence of one another, ${ }^{15}$ especially in light of the established role of blebbing in many immunological disorders. ${ }^{16}$ Another process that involves simultaneously geometries similar to blebs, and cytoskeletal remodelling is spine dynamics. Dendritic spines are submicron-size protrusions on neuronal dendrites, at the receiving end of synaptic transmission. While actin filaments were widely accepted as the only cytoskeletal structural protein hosted in spines, relatively recent advances have also demonstrated the additional presence of microtubules dynamics, in conjunction with $\mathrm{Ca}^{2+}$ influx. ${ }^{17}$ Again, one could hypothesise a complementary role of spine shaping and enhancement of ionic currents. The recent discovery that spines are electrically isolated from the rest of the dendrites while being populated with sodium channels, in turn thought to boost synaptic potential, ${ }^{18}$ further corroborates this postulate.

As a conclusion, the results of this work not only offer an alternative explanation to the experimentally observed "left-shift", but also, while arguably involving different mechanisms and time scales, open the door to the possibility that membrane shape regulation and ionic signal enhancement can act as independent mechanisms while mutually influencing each other. The unique neuronal membrane 
dynamics observed during migration and electrophysiological activity, in particular, lends itself to such hypothesis. 


\section{Acknowledgments}

The authors acknowledge funding from the European Union's Seventh Framework Programme (FP7 20072013) ERC Grant Agreement No.306587.

Disclosure Statement: No competing financial interests exist.

Author Contributions: M.T.K. and A.J. designed the study. M.T.K. implemented the simulations.

M.T.K. and A.J. wrote the manuscript. 


\section{References}

1. Wang, J. a et al. Membrane trauma and Na+ leak from Nav1.6 channels. Am. J. Physiol. Cell Physiol. 297, C823-C834 (2009).

2. Sakmann, B. \& Neher, E. Patch Clamp Techniques for Studying Ionic Channels in Excitable Membranes. Annu. Rev. Physiol. 46, 455-472 (1984).

3. Boucher, P. A., Joós, B. \& Morris, C. E. Coupled left-shift of Nav channels: Modeling the $\mathrm{Na}+$-loading and dysfunctional excitability of damaged axons. J. Comput. Neurosci. 33, 301319 (2012).

4. Jérusalem, A., García-Grajales, J. A., Merchán-Pérez, A. \& Peña, J. M. A computational model coupling mechanics and electrophysiology in spinal cord injury. Biomech. Model. Mechanobiol. 13, 883-896 (2014).

5. García-Grajales, J. A., Rucabado, G., García-Dopico, A., Peña, J. M. \& Jérusalem, A. Neurite, a finite difference large scale parallel program for the simulation of electrical signal propagation in neurites under mechanical loading. PLoS One 10, (2015).

6. Kwong, M. T., García-Grajales, J. A., Homsi, L., Noels, L. \& Jerusalem, A. 3D finite element formulation for mechanical-electrophysiological coupling in axonopathy. Comput. Methods Appl. Mech. Eng. 346, 1025-1050 (2019).

7. Zhang, Y. \& Hamill, O. P. On the discrepancy between whole-cell and membrane patch mechanosensitivity in Xenopus oocytes. J. Physiol. 523, 101-115 (2000).

8. Suchyna, T. M., Markin, V. S. \& Sachs, F. Biophysics and structure of the patch and the gigaseal. Biophys. J. (2009). doi:10.1016/j.bpj.2009.05.018

9. Zhang, L., Jasa, J., Gazonas, G., Jérusalem, A. \& Negahban, M. Extracting continuum-like deformation and stress from molecular dynamics simulations. Comput. Methods Appl. Mech. Eng. 283, 1010-1031 (2015). 
10. Geuzaine, C. \& Remacle, J. F. Gmsh: A three-dimensional finite element mesh generator with built-in pre- and post-processing facilities. Int J Numer Methods Eng. 79, 1309-1331 (2009).

11. Brackenbury, W. J. et al. Functional reciprocity between $\mathrm{Na}+$ channel Nav1.6 and 1 subunits in the coordinated regulation of excitability and neurite outgrowth. Proc. Natl. Acad. Sci. 107, $2283-2288(2010)$.

12. Schaar, B.T. \& McConnell, S.K. Cytoskeletal coordination during neuronal migration. Proc. Natl. Acad. Sci. 102, 13652-13657 (2005).

13. Cui, K. \& Yuan, X.-B. TRP channels and axon pathfinding. Chapter 4 in TRP ion Channel function in sensory transduction and cellular signaling cascades. Liedtke WB, Heller S, editors. CRC Press/Taylor \& Francis (2007).

14. Samanta, S., Hughes, T.E.T. \& Moiseenkova-Bell, V.Y. Transient receptor potential (TRP) channels. Subcell Biochem. Sci. 87, 141-165 (2018).

15. Charras, G.T. A short history of blebbing. J. Microsc. 231, 466-478 (2008).

16. Khajah, M.A. \& Lugmani, Y.A. Involvement of membrane blebbing in immunological disorders and cancer. Med. Princ. Pract. 25(Suppl 2), 18-27 (2017).

17. Merriam, E.B. et al. Synaptic regulation of microtubule dynamics in dendritic spines by calcium, F-actin and drebrin. J. Neurosci. 33, 16471-16482 (2013).

18. Araya, R., Nikolenko, V., Eisenthal, K.B. \& Yuste, R. Sodium channels amplify spine potentials. Proc. Natl. Acad. Sci. 104, 12347-12352 (2005). 


\section{Figure Legends}

FIG. 1. (a) Voltage clamp model setup and displacement field at $5.65 \mathrm{~ms}$. (b) Voltage gradient field post aspiration at $5.65 \mathrm{~ms}$. (c) Voltage gradient field without aspiration at $5.65 \mathrm{~ms}$.

FIG. 2. Comparison of current flowing out of membrane in the pipette with and without suction for the overall membrane in the patch. The inset is a direct comparison between the two current rise tangents (scaled vertically, translated horizontally).

FIG. 3. Potential changes in the cytoplasmic resistance depending on the state of stress: the resistance $R$ of an element is proportional to the distance travelled by the current $(h)$ and inversely proportional to the area that it is crossing $(A)$; here, $\rho$ is the cytoplasmic resistivity. 


\title{
Ionic current enhancement through localised membrane geometrical deformation
}

\author{
Man Ting Kwong ${ }^{1}$, Antoine Jérusalem ${ }^{1 *}$ \\ ${ }^{1}$ Department of Engineering Science, University of Oxford, Parks Road, Oxford OX1 3PJ, UK
}

\author{
*Corresponding author \\ Professor Antoine Jérusalem \\ Address: Department of Engineering Science, University of Oxford, Parks Road, Oxford OX1 3PJ, UK \\ Email: antoine.jerusalem@eng.ox.ac.uk
}

\begin{abstract}
Bleb-inducing aspiration in a patch-clamp has previously been proposed as a controlled proxy for ion channel damage characterisation in brain trauma. Under increasing pressure, voltage gated sodium channels $\mathrm{Na}_{\mathrm{V}} 1.6$ have been observed to exhibit an altered dynamics known as "left-shift". While many numerical models have since been proposed to simulate this behaviour through modification of the ion channel dynamics in the Hodgkin-Huxley model, the effect of the deformed membrane geometry on the electrophysiology has not been explored. Here we reproduce in silico an idealised voltage clamp under the range of pressure used in its in vitro counterparts. The findings show that the geometrical alteration of the membrane within the pipette directly affects the patch electrophysiological measurements without requiring the ion channels to be damaged. This work highlights the need to consider geometrical deformation of the membrane when studying ion channel dynamics in deformed membrane and offers alternative explanations on the effect of blebbing on cellular and brain functions. It also opens the door to the possibility that membrane shape regulation and ionic signal enhancement can act independently with the former influencing the latter in neuronal migration and electrophysiological activity.
\end{abstract}

Keywords: patch-clamp, left-shift, Hodgkin-Huxley, finite element method, electrophysiologicalmechanical coupling 
Patch clamping is an experimental technique, where a patch of cell membrane is drawn into a pipette and its electrophysiology is measured by use of a stimulating electrode within the pipette. In a voltage clamp technique, the electrode inside the pipette is used to stabilise the membrane potential at a fixed voltage, while simultaneously recording the current that flows within the pipette. In particular, when the membrane is not ruptured ("cell attached" configuration), this technique is measuring the electrophysiology of a small number of ion channels within the patch (as opposed to "whole cell" configuration where the membrane inside the pipette is ruptured to record ion channels currents for the entire cell) and the measured feedback current is directly related to their activity.

Cell attached voltage clamp was used by Wang et al. to probe the channel dynamics of $\mathrm{Na}_{\mathrm{V}} 1.6$ in human embryonic kidney cells post-membrane trauma. ${ }^{1}$ Gigaseal (a seal formed between the cell membrane and the pipette wall, reaching resistance in the gigaohms range ${ }^{2}$ ) was achieved by "pre-stretching" patches at suction pressures between -4 and $-7 \mathrm{mmHg}$. Electrophysiological measurements in this configuration were taken as the reference. Subsequently, increasingly higher pressures were applied to the patch, with electrophysiological measurements reported to cause faster rise times in the voltage gated sodium channels $\mathrm{Na}_{\mathrm{V}} 1.6$ gating dynamics: the so-called "left-shift". ${ }^{1}$ Wang et al. hypothesised that direct membrane stretch through pipette suction causes $\mathrm{Na}_{\mathrm{v}} 1.6$ to become "leaky", suggesting that the channels are susceptible to trauma. This work has since motivated the development of many models built on the alteration of the ion channel dynamics in the Hodgkin-Huxley model. ${ }^{3-6}$

Cell membranes are known to deform under applied pressures similarly to two-dimensional fluids, ${ }^{7,8}$ effectively damping an overall membrane deformation to a fraction of its strain at the transmembrane protein level when the protein is untethered, both from the cytoskeleton and the extracellular matrix. ${ }^{9}$ This and the absence of evidence that the electrophysiology cannot be influenced by an alteration of the shape of the membrane patch call into question whether the observed "left-shift" can also occur in the absence of ion channel damage. To this end, a "pre-stretched" membrane patch geometry was modelled in silico by means of a finite element program to simulate the effect of subsequent pipette pressure on the patch electrophysiology. 
The cell geometry within the pipette was represented by a frustum of a cone (with a height of $2 \mu \mathrm{m}$, and upper and lower radii of 0.75 and $0.5 \mu \mathrm{m}$, respectively) and the main cell body immediately beneath the pipette was represented by a $3 \times 1 \times 3 \mu \mathrm{m}^{3}$ rectangular parallelepiped, see Figure 1 . The resulting geometry was discretised with 4,563 quadratic tetrahedral elements. A pressure boundary condition of $-50 \mathrm{mmHg}$ was applied to the top face of the frustum to simulate the suction applied by the pipette, see Figure 1a. The pipette walls and free surfaces of the box domain below were fixed in displacements. Since the electrophysiological-mechanical coupling is solely linked to the geometrical deformation of the membrane (see next paragraph), a linear elastic material law with a Young's modulus, density and Poisson's ratio of $165 \mathrm{kPa}, 993 \mathrm{~kg} \mathrm{~m}^{-3}$ and 0.3 , respectively, ${ }^{6}$ was considered adequate. In the simulations, the applied pressure was kept during action potential (AP) propagation, thus fixing the membrane geometry during the entire electrophysiological measurement. Membrane creep during patch clamp experiments has been previously observed, ${ }^{8}$ however the time scale of the creep is more than an order of magnitude larger than the time scale of an AP. Furthermore, this study focusses on the electrophysiology propagation on different static geometries arising from a pressure of $-50 \mathrm{mmHg}$, therefore a fixed deformed geometry was deemed acceptable.

The open source finite element platform $\mathrm{Gmsh}^{10}$ enriched with a dedicated electrophysiologicalmechanical solver ${ }^{6}$ was used to solve the finite element problem. In this novel approach, the active Hodgkin-Huxley electrophysiological model is imposed directly in the weak form as a Neumann boundary condition on the conductive three-dimensional domain. See Ref. (6) for more details, and in particular, for the values of the model parameters and their expressions in terms of patch area and other geometrical properties. In the work proposed here, regular Hodgkin-Huxley boundary conditions were considered for the membrane surrounding the pipette while the cell attached voltage-clamp at the top face of the frustum (inside the pipette) was modelled through a modified Hodgkin-Huxley boundary condition to include an external voltage of $20 \mathrm{mV}$ for the entire duration $(12 \mathrm{~ms})$ of the simulation so as to trigger an AP in the blebbed membrane within the pipette. The overall model thus remains the same as in Ref. (6), except for the following modification in the Hodgkin-Huxley equation where the term $\Delta \mathrm{V}=20 \mathrm{mV}$ is added: 


$$
i_{n}=c_{m} \frac{\partial V}{\partial t}+g_{N a}\left(V-E_{N a}+\Delta V\right)+g_{K}\left(V-E_{K}+\Delta V\right)+g_{L}\left(V-E_{L}+\Delta V\right)
$$

where $i_{n}, c_{m}, g_{N a}, g_{K}, g L, E_{N a}, E_{K}$ and $E_{L}$ are the outward current to the membrane, the membrane capacitance (for a given area), its $\mathrm{Na}_{\mathrm{v}}$ ion channel, $\mathrm{K}_{\mathrm{v}}$ ion channel and loss conductances, and the corresponding reversal potentials, respectively. Even after deformation, $\mathrm{Na}_{\mathrm{V}}$ and voltage gated potassium channel $\mathrm{K}_{\mathrm{V}}$ conductances were calculated with respect to the undeformed configuration, i.e., the current conductance associated to these channels remained the same throughout the deformation and independently of the area increase of the membrane (said otherwise, the number of ion channels in the top surface of the frustum remains the same). On the other hand, the leak and membrane resistances and capacitances were calculated with respect to the deformed reference frame and thus vary with the increase of surface area. The domain representing the gigasealed membrane (where the membrane is in contact with the pipette) was set to have no electrical flux across it, while the distal outer faces of the cytoplasm away from the pipette were free of flow. The Poisson's equation was chosen to govern the charge distribution in the bulk of the cytoplasm with a cytoplasm resistivity of $1.87 \Omega \mathrm{m} .^{6}$

Figures $1 \mathrm{a}$ and $1 \mathrm{~b}$ show the resultant deformation field at $5.65 \mathrm{~ms}$ and the post-suction intracellular voltage gradient (plotted in the undeformed configuration), respectively, while Figure 1c shows for comparison the voltage gradient at the same time $(5.65 \mathrm{~ms})$ when no suction is applied. Figure 2 shows the time evolution of the overall membrane current outflow in the pipette for both cases, and the respective (shifted) current rise tangents. These results indicate that: i) the geometry of the bleb has a strong influence on the current in the pipette (Figure 1b), and ii) the model is able to simulate some of the characteristics of a "left-shift" behaviour after aspiration without altering the ion channel dynamics, i.e., larger current amplitude and faster rise.

As the area of the membrane increases under negative pressure, a higher overall conductance across the membrane, and thus a faster rise for channel activation can indeed be expected. However, the overall conductance of a cytoplasmic element can either decrease or increase with the deformation, depending whether the current needs to go through more or less material over a larger or smaller area, see Figure 
3. This delicate balance between gain and loss in electrophysiological activity comes off here as an increase of overall current.

It must be emphasised that the magnitude of current in the above simulations is an order of magnitude smaller than the ones reported in the experiments. A simple reason for the discrepancy is the fact that the original Hodgkin-Huxley model was used in this model as a first approximation. In the experiments, a different cell was used and the ion channels were artificially transfected, making the identification of exact model to be used for the electrophysiology challenging. ${ }^{1}$ However, as long as a similar sodium ion channel behaviour is expected, the model's results are expected to qualitatively hold. Finally, the experiments were conducted on cells with $\mathrm{Na}_{\mathrm{v}} 1.6$ with and without auxiliary $\beta 1$ subunit (known to play a role in mechanotransduction behaviour ${ }^{11}$ ) with no marked resulting difference reported between the two, indicating that the reported effect may not be of a mechanosensitive nature. While this a priori seems to reinforce the experimental conclusion that the ion channels dynamics are altered through direct damage, this is also in agreement with the prediction of this work that the membrane deformation itself might be a vector for similar "left-shift" behaviour, independently of the ion channels. Finally, the results of Wang et al. exhibiting a given degree of irreversibility in the alteration of the electrophysiological behaviour ${ }^{1}$ are compatible with the notion of viscoelastic behaviour of the cell during suction, whereby the irreversible geometrical deformation of the membrane would invariably lead to an irreversible electrophysiological alteration in the proposed model. This phenomenon could thus readily be implemented with the right material constitutive model.

The above simulations demonstrate that geometrical alterations can alter the electrophysiological conduction in terms of a decreased rise time of channel activation, increase of current amplitude and signal delay, consistent with the experimentally observed "left-shift", but without the need to explicitly alter the channel gating dynamics. Wang et al.'s conclusions suggested that the "left-shift" dynamics could be justified by an increase in channel opening probability or area, as this would solely result in an increase in current amplitude and that the data thus pointed to trauma-induced $\mathrm{Na}_{\mathrm{V}} 1.6$ dynamics changes ${ }^{1}$. We demonstrate here that, in theory, "left-shift" characteristics can be observed without inducing ion channel damage through the additional consideration of the neighbouring deformed 
membrane. When widening the conclusions of this work to other channels, this result has direct implications on many brain functions.

Neuronal migration during brain development involves a complex coordination of cytoskeletal alterations (involving, among others, actin filaments, microtubules, myosin). ${ }^{12}$ In particular the distal end of the axon, the growth cone, is actively reshaping itself using motile filipodia and lamellipodia to sense its immediate environment. This sensing is done, among others, by use of transient receptor potential channels (TRP) permeable to $\mathrm{Ca}^{2+} \cdot{ }^{13}$ While TRP channels seem to be involved in mechanosensitive gating mechanisms, their exact involvements in neuronal migration is still under investigation. ${ }^{14}$ The result of this work suggests however that a radical change of membrane geometry is enough to affect the behaviour of ionic exchange with the cytoplasm. It is thus possible that the remodelling of the growth cone through F-actin and integrin activities could indirectly affect TRP gating related $\mathrm{Ca}^{2+}$ influx by membrane remodelling. This hypothesis is of particular relevance to the debate over whether blebbing or $\mathrm{Ca}^{2+}$ influx (commonly observed conjointly) is the cause or consequence of one another ${ }^{15}$ especially in light of the established role of blebbing in many immunological disorders. ${ }^{16}$ Another process that involves simultaneously geometries similar to blebs, and cytoskeletal remodelling is spine dynamics. Dendritic spines are submicron-size protrusions on neuronal dendrites, at the receiving end of synaptic transmission. While actin filaments were widely accepted as the only cytoskeletal structural protein hosted in spines, relatively recent advances have also demonstrated the additional presence of microtubules dynamics, in conjunction with $\mathrm{Ca}^{2+}$ influx. ${ }^{17}$ Again, one could hypothesise a complementary role of spine shaping and enhancement of ionic currents. The recent discovery that spines are electrically isolated from the rest of the dendrites while being populated with sodium channels, in turn thought to boost synaptic potential, ${ }^{18}$ further corroborates this postulate.

As a conclusion, the results of this work not only offer an alternative explanation to the experimentally observed "left-shift", but also, while arguably involving different mechanisms and time scales, open the door to the possibility that membrane shape regulation and ionic signal enhancement can act as independent mechanisms while mutually influencing each other. The unique neuronal membrane 
dynamics observed during migration and electrophysiological activity, in particular, lends itself to such hypothesis. 


\section{Acknowledgments}

The authors acknowledge funding from the European Union's Seventh Framework Programme (FP7 20072013) ERC Grant Agreement No.306587.

Disclosure Statement: No competing financial interests exist.

Author Contributions: M.T.K. and A.J. designed the study. M.T.K. implemented the simulations. M.T.K. and A.J. wrote the manuscript. 


\section{References}

1. Wang, J. a et al. Membrane trauma and Na+ leak from Nav1.6 channels. Am. J. Physiol. Cell Physiol. 297, C823-C834 (2009).

2. Sakmann, B. \& Neher, E. Patch Clamp Techniques for Studying Ionic Channels in Excitable Membranes. Annu. Rev. Physiol. 46, 455-472 (1984).

3. Boucher, P. A., Joós, B. \& Morris, C. E. Coupled left-shift of Nav channels: Modeling the $\mathrm{Na}+-$ loading and dysfunctional excitability of damaged axons. J. Comput. Neurosci. 33, 301319 (2012).

4. Jérusalem, A., García-Grajales, J. A., Merchán-Pérez, A. \& Peña, J. M. A computational model coupling mechanics and electrophysiology in spinal cord injury. Biomech. Model. Mechanobiol. 13, 883-896 (2014).

5. García-Grajales, J. A., Rucabado, G., García-Dopico, A., Peña, J. M. \& Jérusalem, A. Neurite, a finite difference large scale parallel program for the simulation of electrical signal propagation in neurites under mechanical loading. PLoS One 10, (2015).

6. Kwong, M. T., García-Grajales, J. A., Homsi, L., Noels, L. \& Jerusalem, A. 3D finite element formulation for mechanical-electrophysiological coupling in axonopathy. Comput. Methods Appl. Mech. Eng. 346, 1025-1050 (2019).

7. Zhang, Y. \& Hamill, O. P. On the discrepancy between whole-cell and membrane patch mechanosensitivity in Xenopus oocytes. J. Physiol. 523, 101-115 (2000).

8. Suchyna, T. M., Markin, V. S. \& Sachs, F. Biophysics and structure of the patch and the gigaseal. Biophys. J. (2009). doi:10.1016/j.bpj.2009.05.018

9. Zhang, L., Jasa, J., Gazonas, G., Jérusalem, A. \& Negahban, M. Extracting continuum-like deformation and stress from molecular dynamics simulations. Comput. Methods Appl. Mech. Eng. 283, 1010-1031 (2015). 
10. Geuzaine, C. \& Remacle, J. F. Gmsh: A three-dimensional finite element mesh generator with built-in pre- and post-processing facilities. Int J Numer Methods Eng. 79, 1309-1331 (2009).

11. Brackenbury, W. J. et al. Functional reciprocity between $\mathrm{Na}+$ channel Nav1.6 and 1 subunits in the coordinated regulation of excitability and neurite outgrowth. Proc. Natl. Acad. Sci.107, 2283-2288 (2010).

12. Schaar, B.T. \& McConnell, S.K. Cytoskeletal coordination during neuronal migration. Proc. Natl. Acad. Sci. 102, 13652-13657 (2005).

13. Cui, K. \& Yuan, X.-B. TRP channels and axon pathfinding. Chapter 4 in TRP ion Channel function in sensory transduction and cellular signaling cascades. Liedtke WB, Heller S, editors. CRC Press/Taylor \& Francis (2007).

14. Samanta, S., Hughes, T.E.T. \& Moiseenkova-Bell, V.Y. Transient receptor potential (TRP) channels. Subcell Biochem. Sci. 87, 141-165 (2018).

15. Charras, G.T. A short history of blebbing. J. Microsc. 231, 466-478 (2008).

16. Khajah, M.A. \& Lugmani, Y.A. Involvement of membrane blebbing in immunological disorders and cancer. Med. Princ. Pract. 25(Suppl 2), 18-27 (2017).

17. Merriam, E.B. et al. Synaptic regulation of microtubule dynamics in dendritic spines by calcium, F-actin and drebrin. J. Neurosci. 33, 16471-16482 (2013).

18. Araya, R., Nikolenko, V., Eisenthal, K.B. \& Yuste, R. Sodium channels amplify spine potentials. Proc. Natl. Acad. Sci. 104, 12347-12352 (2005). 


\section{Figure Legends}

FIG. 1. (a) Voltage clamp model setup and displacement field at $5.65 \mathrm{~ms}$. (b) Voltage gradient field post aspiration at $5.65 \mathrm{~ms}$. (c) Voltage gradient field without aspiration at $5.65 \mathrm{~ms}$.

FIG. 2. Comparison of current flowing out of membrane in the pipette with and without suction for the overall membrane in the patch. The inset is a direct comparison between the two current rise tangents (scaled vertically, translated horizontally).

FIG. 3. Potential changes in the cytoplasmic resistance depending on the state of stress: the resistance $R$ of an element is proportional to the distance travelled by the current $(h)$ and inversely proportional to the area that it is crossing $(A)$; here, $\rho$ is the cytoplasmic resistivity. 
FIG. 1 (c)

\section{Voltage gradient $\left(\mathrm{Vm}^{-1}\right)$}




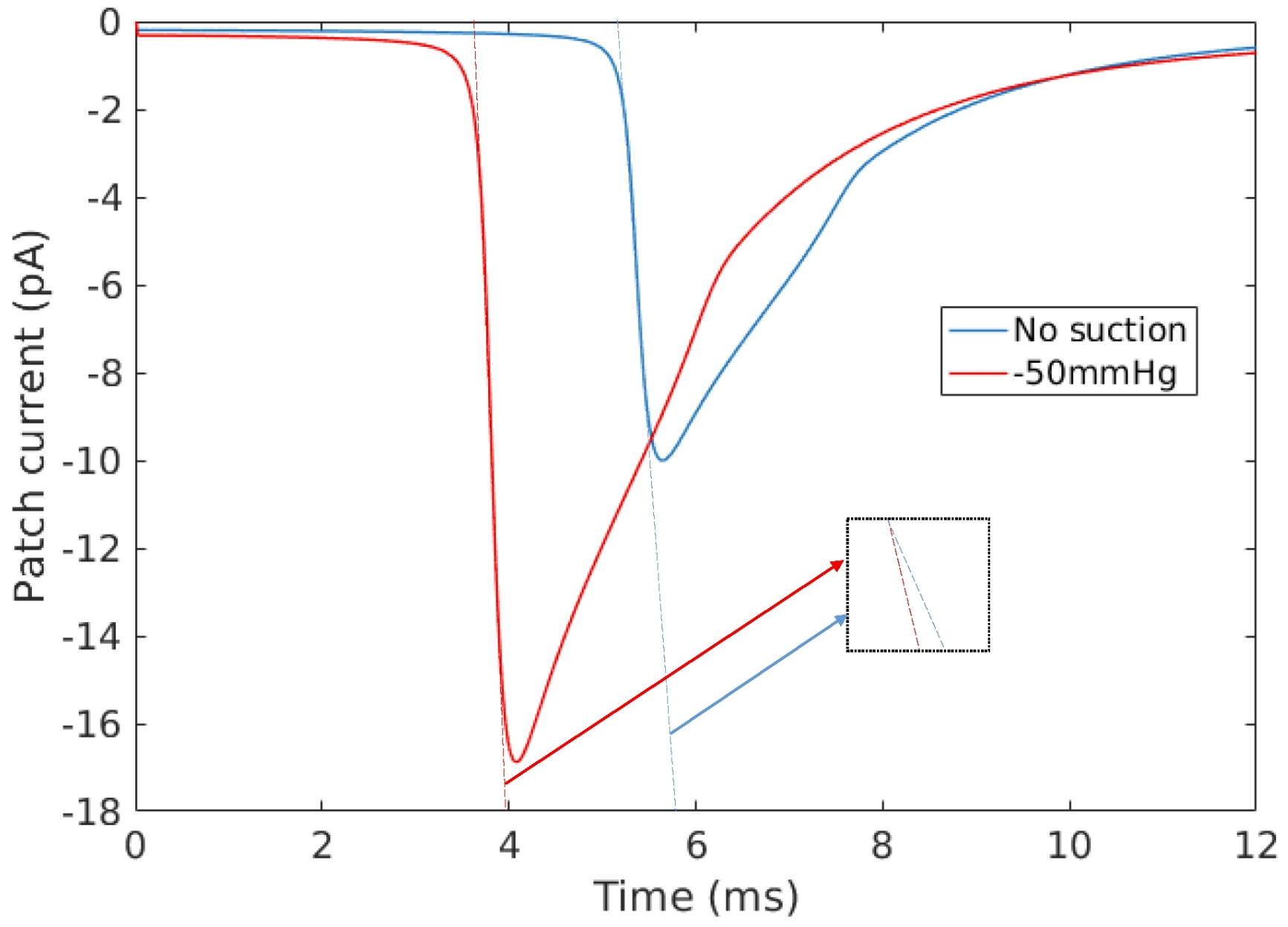

